\title{
Matching the Quasi Parton Distribution in a Momentum Subtraction Scheme
}

\section{Yong Zhao*}

Center for Theoretical Physics, Massachusetts Institute of Technology, Cambridge, MA 02139, USA

E-mail: yzhaoqcdemit.edu

\section{lain W. Stewart}

Center for Theoretical Physics, Massachusetts Institute of Technology, Cambridge, MA 02139, USA

E-mail: iains@mit.edu

\begin{abstract}
The quasi parton distribution is a space-like correlation of quarks and gluons along the $z$ direction in a nucleon which can be calculated directly from lattice QCD. At large but finite nucleon momentum, the quasi parton distribution can be perturbatively matched to the physical parton distribution function through a factorization formula in large momentum effective theory. Following a nonperturbative renormalization of the quasi parton distribution in a regularization independent momentum subtraction scheme, we establish its matching to the $\overline{\mathrm{MS}}$ PDF and calculate the non-singlet matching coefficient at next-to-leading order in perturbation theory.
\end{abstract}

QCD Evolution 2017

22-26 May, 2017

Jefferson Lab Newport News, VA - USA

\footnotetext{
* Speaker.
} 


\section{Introduction}

PDFs are the most basic and important objects for us to understand hadron structures in modern physics. Since PDFs are intrinsic properties of the hadron that include low energy degrees of freedom, they can only be calculated with nonperturbative methods such as lattice QCD. Up to now, our most precise knowledge about PDFs comes from global fits to experimental data [1, 2, 3, 4, 5], despite that their low-order moments have been directly calculated with lattice methods $[6,7,8]$,

In parton physics, PDFs are defined as the nucleon matrix elements of light-cone correlation operators. For example, in dimensional regularization with $d=4-2 \varepsilon$, the bare unpolarized quark distribution function is

$$
q_{i}(x, \varepsilon) \equiv \int \frac{d \xi^{-}}{4 \pi} e^{-i x P^{+} \xi^{-}}\left\langle P\left|\bar{\psi}_{i}\left(\xi^{-}\right) \gamma^{+} W\left(\xi^{-}, 0\right) \psi_{i}(0)\right| P\right\rangle,
$$

where $x$ is the momentum fraction, $i$ is a flavor index, the nucleon momentum $P^{\mu}=\left(P^{0}, 0,0, P^{z}\right)$, $\xi^{ \pm}=(t \pm z) / \sqrt{2}$ are the light-cone coordinates, and the Wilson line $W$ is given by the path-ordered exponential

$$
W\left(\xi^{-}, 0\right)=P \exp \left(-i g \int_{0}^{\xi^{-}} d \eta^{-} A^{+}\left(\eta^{-}\right)\right)
$$

The renormalized PDFs $q_{j}(y, \mu)$ are defined in the $\overline{\mathrm{MS}}$ scheme as

$$
q_{i}(x, \varepsilon)=\sum_{j} \int_{x}^{1} \frac{d y}{y} Z_{i j}^{\overline{\mathrm{MS}}}\left(\frac{x}{y}, \varepsilon, \mu\right) q_{j}(y, \mu),
$$

$Z^{\overline{\mathrm{MS}}}$ is a function of $x / y$, the flavor indices $i, j$ include the gluon, and $\mu$ is the renormalization scale. The $\overline{\mathrm{MS}}$ definition of the PDF has an interpretation as a parton number density in the lightcone gauge $A^{+}=0$, and is the most widely used definition for the PDF in factorization theorems. The dependence of the PDF correlator on the light-cone makes it essentially impossible to directly calculate them using lattice QCD in Euclidean space with imaginary time.

In Ref. [9], Ji proposed that instead of calculating light-cone correlations, one can start from a spatial correlation — called a quasi-PDF — which can be calculated in lattice QCD. The bare quasiPDF is defined in momentum space $(x)$ and coordinate space $(z)$ as

$$
\begin{aligned}
& \tilde{q}\left(x, P^{z}, \varepsilon\right) \equiv \int_{-\infty}^{\infty} \frac{d z}{2 \pi} e^{i x P^{z} z} \tilde{q}_{i}^{B}\left(z, P^{z}, \varepsilon\right), \\
& \tilde{q}_{i}\left(z, P^{z}, \varepsilon\right) \equiv \frac{1}{2}\left\langle P\left|\bar{\psi}(z) \gamma^{z} W_{z}(z, 0) \psi(0)\right| P\right\rangle,
\end{aligned}
$$

where the spacelike Wilson line is

$$
W_{z}(z, 0)=P \exp \left(-i g \int_{0}^{z} d z^{\prime} A^{z}\left(z^{\prime}\right)\right) .
$$

Unlike the PDF in Eq. (1.1) that is invariant under a boost along the $z$ direction, the quasi-PDF changes dynamically under such a boost, which is encoded by its nontrivial dependence on the 
nucleon momentum $P^{z}$. The quasi-PDF in coordinate space $\tilde{q}_{j}\left(z, P^{z}, \tilde{\mu}\right)$ is multiplicatively renormalized in dimensional regularization, so we can write

$$
\tilde{q}_{i}\left(z, P^{z}, \varepsilon\right)=\tilde{Z}_{i}^{X}\left(z, P^{z}, \varepsilon, \tilde{\mu}\right) \tilde{q}_{i}^{X}\left(z, P^{z}, \tilde{\mu}\right) .
$$

Here the position space renormalization factors $\tilde{Z}_{i}^{X}(z, \varepsilon, \tilde{\mu})$ are defined in a particular scheme $X$, such as $\overline{\mathrm{MS}}$, a momentum-subtraction scheme etc., and $\tilde{\mu}$ is a renormalization scale for the quasiPDF (whose definition also depends on the scheme $X$ ). Fourier transforming to momentum space as in Eq. (1.4), the renormalization for the quasi-PDF involves a convolution in the momentum fraction,

$$
\tilde{q}_{i}\left(x, P^{z}, \varepsilon\right)=P^{z} \int_{-\infty}^{+\infty} d x^{\prime} \tilde{Z}_{i}^{X}\left(x-x^{\prime}, P^{z}, \varepsilon, \tilde{\mu}\right) \tilde{q}_{i}^{X}\left(x^{\prime}, P^{z}, \tilde{\mu}\right)
$$

The structure of the renormalization of the quasi-PDF in Eqs. (1.6) and (1.7) is similar to that of the quark beam-function $[10,11]$, which is a proton distribution with separations along both the plus and minus light-cone directions. Ref. [11] gives an all orders proof of the position space multiplicative renormalization of the beam function, and this proof also implied that $Z_{i j} \propto \delta_{i j}$, so there is never parton mixing in this case. In dimensional regularization it has been explicitly demonstrated that the quasi-PDF is multiplicatively renormalized to two loops [12]. Recently a proof of the multiplicative renormalization has been given both non-perturbatively in Ref. [13] and diagrammatically in Ref. [14]. The multiplicative renormalization property present in Eq. (1.6) essentially follows from the known renormalization structure of QCD and of Wilson lines. Ref. [14] has also demonstrated that there is no flavor mixing in the renormalization of the quasi-PDF.

For a nucleon moving with finite but large momentum $P^{z} \gg \Lambda_{\mathrm{QCD}}$, the quasi-PDF can be matched onto the PDF through a momentum space factorization formula $[9,15]:{ }^{1}$

$$
\begin{aligned}
\tilde{q}_{i}^{X}\left(x, P^{z}, \tilde{\mu}\right)= & \int_{-1}^{+1} \frac{d y}{|y|} C_{i j}^{X}\left(\frac{x}{y}, \frac{\tilde{\mu}}{P^{z}}, \frac{\mu}{|y| P^{z}}\right) q_{j}(y, \mu) \\
& +\mathscr{O}\left(\frac{M^{2}}{P_{z}^{2}}, \frac{\Lambda_{\mathrm{QCD}}^{2}}{P_{z}^{2}}\right),
\end{aligned}
$$

where $C_{i j}$ is the matching coefficient, and the $\mathscr{O}\left(M^{2} / P_{z}^{2}, \Lambda_{\mathrm{QCD}}^{2} / P_{z}^{2}\right)$ terms are higher-twist corrections suppressed by the nucleon momentum ( $M$ is the nucleon mass). Here $q_{j}(y, \mu)$ for negative $y$ corresponds to the anti-quark contribution. Note that the matching coefficient depends on the quasi-PDF scheme choice $X$, and that for $q_{j}(y, \mu)$ we always assume the $\overline{\mathrm{MS}}$ scheme. Both sides of Eq. (1.8) are formally $\mu$ independent, but both do depend on the scale $\tilde{\mu}$ for the renormalized quasi-PDF, and this dependence need not be small. The indicated power corrections are related to higher-twist contributions in the quasi-PDF. Note that it is important to distinguish between the renormalization of the PDF and quasi-PDF given by the $Z_{i j} \mathrm{~s}$ and $\tilde{Z}_{i j} \mathrm{~s}$, and the matching coefficients given by the $C_{i j}$ s. The renormalization constants occur in a relation between bare and renormalized matrix elements for the same operators. On the other hand the matching coefficients occur in a relation between renormalized matrix elements of different operators. The $\tilde{q}$ and $q$ have the same

\footnotetext{
${ }^{1}$ In this formula we write $\mu /|y| P_{z}$ for the third argument of the matching coefficient. This has recently been proven to be the correct result in Ref. [16].
} 
infrared (IR) divergences (which are collinear divergences in Minkowski space), and at perturbative scales $\mu$ and $\tilde{\mu}$ the $C_{i j}$ s can be calculated order by order in $\alpha_{s}$.

Based on Ji's proposal, the procedure of calculating PDF from lattice QCD can be summarized as:

1. Lattice simulation of the quasi-PDF;

2. Renormalization of the quasi-PDF in a particular scheme on the lattice;

3. Subtraction of higher-twist corrections;

4. Matching quasi-PDF in the particular scheme to PDF in the $\overline{\mathrm{MS}}$ scheme.

Efforts have been made to use this proposal to calculate the iso-vector quark distributions $f_{u-d}$, including unpolarized, polarized, and transversity distributions, as well as pion distribution amplitude, from lattice QCD [17, 18, 19, 20, 21, 22, 23, 24]. For this channel the mixing is not important and so the indicies $i$ and $j$ are dropped. The one-loop matching coefficients were first calculated in a transverse momentum cutoff scheme in Ref. [25], which we denote as $C^{\Lambda_{T}}\left(x, \Lambda_{T} / P^{z}, \mu / p^{z}\right)$. The result was confirmed in Refs. [26, 18]. The nucleon-mass corrections of $O\left(M^{2} / P_{z}^{2}\right)$ have already been included in the lattice calculations [17, 18, 19, 20, 21, 23, 24], and the $O\left(\Lambda_{\mathrm{QCD}}^{2} / P_{z}^{2}\right)$ correction was numerically fitted in Ref. [19]. (A direct lattice calculation of the $O\left(\Lambda_{\mathrm{QCD}}^{2} / P_{z}^{2}\right)$ correction is still desired from the theoretical point of view). In the analyses of Refs $[17,18,19,20]$ the renormalization of the lattice matrix element of quasi-PDF, i.e., Step 2, was absent. With increasing nucleon momentum $P^{z}$, lattice renormalization will be the most important factor that limits the precision of the calculation of PDFs. Recently, renormalization of the quasi-PDF has been considered Refs [27, 28, 29, 30, 22, 21, 23, 31, 13, 24]. In other recent work, a related pseudo-PDF distribution was defined [32], which has been studied in [33, 34, 35].

One of the standard methods to renormalize operators in lattice QCD is the lattice perturbation theory [36]. The perturbative renormalization of the quasi-PDF at one-loop order has recently become available for the Wilson-Clover action [29, 30]. In practice, it requires a significant amount of work to compute lattice Feynman diagrams for the quasi-PDF, which limits the ability to go to higher loop orders, and thus the precision that can be achieved. Moreover, fixed-order perturbative renormalization is not reliable when the operator suffers from power divergences under lattice regularization. An alternative is nonperturbative methods, such as the regularization-invariant momentum subtraction (RI/MOM) scheme, that has been widely used to renormalize local operators on the lattice [37]. Work in progress to calculate the lattice quasi-PDFs in the RI/MOM scheme has been reported in $[22,23,31,24]$, and appears to be the most promising route for future higher precision quasi-PDF determinations. $^{2}$

In this paper we focus on Step 4 when the lattice quasi-PDF is defined in the RI/MOM scheme, the details of which have been elaborated in Ref. [38] and applied to the analysis of Ref. [23]. In particular we carry out a perturbative calculation of the matching coefficient that enables this lattice quasi-PDF to be directly matched onto the $\overline{\mathrm{MS}} \mathrm{PDF}$. We denote this matching coefficient by $C^{\mathrm{OM}}$ and it will depend on the scale of the off-shell subtraction $\mu_{R}$. The renormalized matrix elements

\footnotetext{
${ }^{2}$ Indeed, the result for the matching coefficient computed in this paper has already been used in the lattice calculation in Ref. [23].
} 
in the RI/MOM scheme are independent of the UV regularization, so we carry out this matching perturbatively with dimensional regularization. Our result for the matching coefficient $C^{\mathrm{OM}}$ also exhibits insensitivity to a UV cutoff in the integral in Eq. (1.8), $|y|<y_{\text {cut }}$, unlike the earlier result for $C^{\Lambda_{T}}$. As a biproduct of our calculations, we also determine $C^{\overline{\mathrm{MS}}}$, which is the matching coefficient to go between the $\overline{\mathrm{MS}}$ quasi-PDF and $\overline{\mathrm{MS}} \mathrm{PDF}$. We find that $C^{\overline{\mathrm{MS}}}$ behaves in a similar fashion to $C^{\Lambda_{T}}$.

An alternate to the approach we take here would be to convert the lattice quasi-PDF defined with nonperturbative renormalization in the RI/MOM scheme back to the $\overline{\mathrm{MS}}$ scheme perturbatively [30]. This would then allow the $C^{\overline{\mathrm{MS}}}$ matching result to be used. Our approach is more direct, with only a single step involving a perturbative calculation. Nevertheless it would be interesting to compare both approaches.

In Sec. 2 we elaborate on the procedure of implementing the RI/MOM scheme for the quasiPDF. Then in Sec. 3 we calculate the RI/MOM scheme quasi-PDF and the one-loop matching coefficient between this quasi-PDF and the PDF in the $\overline{\mathrm{MS}}$ scheme. We do this first in Feynman gauge and then in a general covariant gauge. We also give the analogous matching coefficient needed for the $\overline{\mathrm{MS}}$ quasi-PDF, and quote the known result for the transverse cutoff quasi-PDF for comparison. In Sec. 4 we analyze these results and give a numerical comparison between the quasi-PDF obtained with the matching coefficients and the PDF. We conclude in Sec. 5.

\section{Renormalization of Quasi PDF in the RI/MOM Scheme}

In the RI/MOM scheme we will define the renormalization constant $\tilde{Z}^{\mathrm{OM}}\left(z, p_{R}^{z}, \Lambda, \mu_{R}\right)$ in Eq. (1.6) by imposing a condition on the quasi-PDF evaluated with massless quark states $|p s\rangle$ of momentum $p$ and spin $s$ with $p^{2} \neq 0$. We study the iso-vector (non-singlet) case, so that we do not have to consider operator mixing. Here the RI/MOM renormalization condition is

$$
\begin{aligned}
& \left.\tilde{Z}^{\mathrm{OM}}\left(z, p_{R}^{z}, \Lambda, \mu_{R}\right)^{-1} \sum_{s}\left\langle p s\left|\bar{\psi}(z) \gamma^{z} W(z, 0) \psi(0)\right| p s\right\rangle\right|_{\begin{array}{c}
p^{2}=-\mu_{R}^{2} \\
p^{z}=p_{R}^{2}
\end{array}} \\
& =\left.\sum_{s}\left\langle p s\left|\bar{\psi}(z) \gamma^{z} W(z, 0) \psi(0)\right| p s\right\rangle\right|_{\text {tree }} \\
& =\left.4 p^{z} e^{-i z p^{z}} \zeta\right|_{p^{z}=p_{R}^{z}},
\end{aligned}
$$

where in general $p^{\mu}=\left(p^{0}, 0,0, p^{z}\right)$,

$$
\zeta=\frac{1}{4 p^{z}} \sum_{s} \bar{u}^{s} \gamma^{z} u^{s}
$$

contains the non-singlet spinor factor and the sum over spins $s$, and $\mu_{R}$ is the renormalization scale. In Eq. (2.1) $\Lambda$ denotes the UV cutoff and this definition applies for both lattice QCD calculations where $\Lambda=a^{-1}$ is the inverse lattice spacing, and for continuum dimensional regularization calculations where $\Lambda=\varepsilon$. In addition to fixing $p^{2}=-\mu_{R}^{2}$, the choice of the momentum $p^{z}$ is also a free parameter that is part of what specifies the scheme, and which, for example, does not have to be equal to the proton momentum $P^{z}$. For this reason we have denoted this dependence in $\tilde{Z}^{\mathrm{OM}}$ by $p_{R}^{z}$. This slightly generalizes Eqs. (1.7) and (1.8) since now there are two arguments $p_{R}^{z}$, and the 
momentum of the state $P^{z}$ appearing in the quasi-PDF matrix element. The factorization formula for matching in Eq. (1.8) with the quasi-PDF in the RI/MOM scheme therefore becomes

$$
\tilde{q}_{i}^{\mathrm{OM}}\left(x, P^{z}, p_{R}^{z}, \mu_{R}\right)=\int_{-1}^{+1} \frac{d y}{|y|} C_{i j}^{\mathrm{OM}}\left(\frac{x}{y}, \frac{\mu_{R}}{p_{R}^{z}}, \frac{\mu}{|y| P^{z}}\right) q_{j}(y, \mu) .
$$

On the lattice the renormalization is imposed with a Euclidean momentum, $p^{2}=-p_{E}^{2}$, and we set $p_{E}^{2}=\mu_{R}^{2}$ in the condition in Eq. (2.1). For an on-shell vector current matrix element the unique Dirac structure would be $\gamma^{z}$, but since the external state is off-shell, the Feynman diagrams will lead to additional structures such as $p^{z} \not p / p^{2}$. To deal with this, we evaluate the external states with Dirac spinors $\bar{u}(p, s)$ and $u(p, s)$ and replace

$$
\sum_{s} \bar{u}(p, s) \Upsilon(z) u(p, s) \rightarrow \operatorname{Tr}[\not p \Upsilon(z)] \zeta,
$$

in order to fully define our off-shell prescription for evaluating the matrix element on the LHS of Eq. (2.1).

An alternate to using Eq. (2.4) would be to reduce the Dirac structure of $\Upsilon$ to a minimal basis of $\left\{\gamma^{z}, \not p\right\}$ terms, and then only utilize the coefficient of the term proportional to $\gamma^{z}$ to define the RI/MOM quasi-PDF,

$$
\left.\sum_{s} \bar{u}(p, s) \Upsilon(z) u(p, s) \rightarrow \Upsilon(z)\right|_{\gamma^{z}} \zeta
$$

This alternate definition of the RI/MOM scheme has been studied in Ref. [38].

A key advantage of using the RI/MOM scheme is that although the renormalization factor $\tilde{Z}^{\mathrm{OM}}$ and bare matrix element depend on the choice of regulator, the renormalized quasi-PDF does not,

$$
\tilde{q}^{\mathrm{OM}}\left(z, P^{z}, p_{R}^{z}, \mu_{R}\right)=\tilde{Z}^{\mathrm{OM}}\left(z, p_{R}^{z}, \Lambda, \mu_{R}\right)^{-1}\left\langle P\left|\bar{\psi}(z) \gamma^{z} W(z, 0) \psi(0)\right| P\right\rangle
$$

where the state $|P\rangle$ may be a proton for the proton quasi-PDF, or a quark for the quark quasi-PDF, etc. Here the $\Lambda$ dependence formally cancels out between $\tilde{Z}^{\mathrm{OM}}$ and the matrix element on the RHS, and the final result for the renormalized quasi-PDF is independent of the choice of UV regulator. For this reason $\tilde{q}^{\mathrm{OM}}\left(z, P^{z}, p_{R}^{z}, \mu_{R}\right)$ is referred to as regulator independent. In practice there may be power suppressed cutoff dependence in the renormalized quasi-PDF due to approximations used for its calculation. For example, on the lattice there are discretization effects of $\mathscr{O}\left(a p^{z}, a \sqrt{p_{E}^{2}}, a \mu_{R}\right)$ which are small in the region $\Lambda_{\mathrm{QCD}} \ll p^{z}, \sqrt{p_{E}^{2}}, \mu_{R} \ll 1 / a$, and only formally vanish in the continuum limit. These effects can be reduced to $O\left(a^{2}\right)$ with improved action methods [37].

In the lattice implementation it should be emphasized that the renormalization constant in Eq. (2.1) is computed in Euclidean space, but still properly renormalizes the Minkowski space matrix elements. The quasi-PDF proton matrix element in Eq. (2.6) is computed as a Minkowskian matrix element, and hence has the same infrared behavior as the standard PDF, a point that is crucial for the matching in Eq. (1.8). Thus the difference between collinear singularities in Minkowski space and the Euclidean space [39] does not affect the quasi-PDF paradigm. This issue has been addressed in detail in Refs [40, 41]. 
As has been shown in Ref. [25], the quasi-PDF has a linear ultraviolet (UV) divergence. The linear divergence arises from the self energy of the finite length space-like Wilson line $W(z, 0)$ in the quasi-PDF, which can be renormalized as [42, 43, 44]

$$
W^{B}(z, 0)=Z_{z} e^{\delta m|z|} W^{R}(z, 0),
$$

where " $\mathrm{B}$ " and " $\mathrm{R}$ " stand for bare and renormalized. The exponential factor $e^{\delta m|z|}$ introduces counterterms that cancel the linear divergences $\delta m \sim \Lambda$, whereas the rest of the renormalization factor $Z_{z}$ depends on the end points of the Wilson line, including the coordinates 0 and $z$, and includes only logarithmic divergences. We can generalize this renormalization relation to gauge-invariant nonlocal quark bilinear operators, as was proven in $[13,14]$, so that the quasi-PDF renormalization can be split into two parts

$$
\tilde{Z}\left(z, p_{R}^{z}, \Lambda, \tilde{\mu}\right)=e^{\delta m|z|} \tilde{Z}_{\psi}\left(z, p_{R}^{z}, \tilde{\mu}\right),
$$

where $\delta m$ contains all linear divergences $\propto \Lambda$, while $\tilde{Z}_{\psi}\left(z, p_{R}^{z}, \tilde{\mu}\right)$ depends on the end points and includes all logarithmic divergences. Both $\delta m$ and $\tilde{Z}_{\psi}$ can in principle depend on the UV cutoff $\Lambda$, renormalization parameter $\tilde{\mu}$, and momentum of the state used to specify the renormalization scheme $p_{R}^{z}$. In order to specify a well defined renormalization scheme with the split in Eq. (2.8), a distinct definition must be given for $\delta m$ and $\tilde{Z}_{\psi}$. It would be useful if we can redefine the quasi-PDF to make it free of linear divergence, such as the treatment for transverse momentum distributions [45, 46], or the gradient flow method [47, 48], but a practical solution on the lattice has not yet been proposed or carried out. With a lattice regulator $\delta m$ will include a linear divergent term $\propto 1 / a$, and properly canceling these linear divergences is important numerically. One strategy is to determine $\delta m$ non-perturbatively from the renormalization of Wilson loop that corresponds to the static quark-antiquark potential [49, 27, 21,31], which has been explicitly verified to cancel the linear divergence in the quasi-PDF at one loop [27].

In the RI/MOM scheme it is not necessary to separately define $\delta m$ and $\tilde{Z}_{\psi}$, and we can simply use $\tilde{Z}^{\mathrm{OM}}$ which includes all divergences. Both linear and logarithmic UV cutoff dependence cancel out in Eq. (2.6), and this cancellation relies only on the multiplicative renormalizability in Eq. (1.6). This appears to be the simplest approach when carrying out a fully nonperturbative renormalization on the lattice. (If $\tilde{Z}_{\psi}(z)$ is calculated in lattice perturbation theory, then it is likely beneficial to separately define and calculate $\delta m$ non-perturbatively.) This also suffices for our purposes for defining the renormalized quasi-PDF, since it is only needed as input for our calculation that matches directly onto the $\overline{\mathrm{MS}} \mathrm{PDF}$ by determining the coefficient $C^{\mathrm{OM}}$ to be used in Eq. (1.8). We exploit the independence of the RI/MOM scheme to the choice of UV regulator to carry out this matching calculation using dimensional regularization.

\section{Matching between quasi-PDF and PDF}

In this section we calculate the one-loop matching coefficient that converts the renormalized quasi-PDF in the RI/MOM scheme to renormalized PDF in the $\overline{\mathrm{MS}}$ scheme. To do the matching, we compare the two matrix elements in an off-shell quark state with momentum $p^{\mu}=\left(p^{0}, 0,0, p^{z}\right)$, and $p^{2}<0$. In dimensional regularization with $d=4-2 \varepsilon$ and an expansion about $\varepsilon=0$, we do 
not see the linear divergence. Although the operator is gauge invariant, its off-shell matrix element is generally not. As a result, the renormalization constant defined in the momentum subtraction scheme will be gauge dependent too. We will start by carrying out this calculation in the Feynman gauge. The result in a general covariant gauge will then be given in Sec. 3.1, which includes the Landau-gauge result that is the most relevant for lattice simulations.

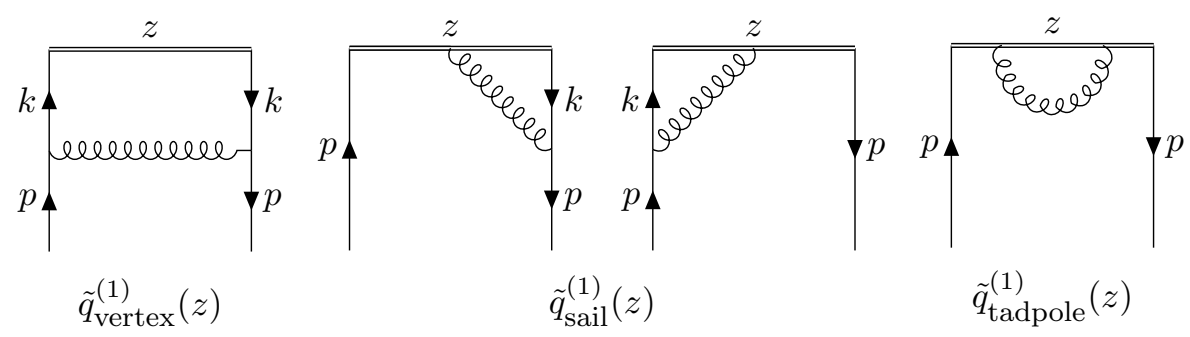

Figure 1: One-loop Feynman diagrams for the quasi-PDF. The standard quark self energy wavefunction renormalization is also included.

Although in this section we carry out the matching using the off-shellness as an IR regulator, the result for the matching coefficient is independent of the choice of IR regulator. Therefore if we carry out the calculation with an on-shell IR regulator like dimensional regularization we will obtain the same matching coefficient between the RI/MOM quasi-PDF and $\overline{\mathrm{MS}}$ PDF.

To define the off-shell quark matrix element we will use the definition in Eq. (2.4). For the quasi-PDF, we use the momentum space Feynman rules for the coordinate space $\tilde{q}\left(z, p^{z}, \varepsilon\right)$ [27]. At tree level we obtain

$$
\tilde{q}_{\text {tree }}^{(0)}\left(z, p^{z}\right)=4 p^{z} \zeta e^{-i z p^{z}}
$$

The one-loop Feynman diagrams are shown in Fig. 1. With the quark wavefunction renormalization corrections, the bare quasi-PDF in the Feynman gauge is [38]

$$
\begin{aligned}
\tilde{q}^{(1)}\left(z, p^{z}, 0, p^{2}\right)= & \frac{\alpha_{s} C_{F}}{2 \pi}\left(4 p^{z} \zeta\right) \int_{-\infty}^{\infty} d x\left(e^{-i x p^{z} z}-e^{-i p^{z} z}\right) \\
& \times \begin{cases}\frac{1}{\sqrt{1-\rho}}\left[\frac{1+x^{2}}{1-x}-\frac{\rho}{2(1-x)}\right] \ln \frac{2 x-1+\sqrt{1-\rho}}{2 x-1-\sqrt{1-\rho}}-\frac{\rho}{4 x(x-1)+\rho}+1 & x>1 \\
\frac{1}{\sqrt{1-\rho}}\left[\frac{1+x^{2}}{1-x}-\frac{\rho}{2(1-x)}\right] \ln \frac{1+\sqrt{1-\rho}}{1-\sqrt{1-\rho}}-\frac{2 x}{1-x} & 0<x<1, \\
\frac{1}{\sqrt{1-\rho}}\left[\frac{1+x^{2}}{1-x}-\frac{\rho}{2(1-x)}\right] \ln \frac{2 x-1-\sqrt{1-\rho}}{2 x-1+\sqrt{1-\rho}}+\frac{\rho}{4 x(x-1)+\rho}-1 & x<0\end{cases}
\end{aligned}
$$

where

$$
\rho \equiv \frac{\left(-p^{2}-i \varepsilon\right)}{p_{z}^{2}}
$$

and the $-i \varepsilon$ allows us to easily analytically continue $\rho$ from $\rho<1$ to $\rho>1$. For $x \rightarrow \pm \infty$ the integrand in Eq. (3.2) is $\propto 1 / x$ and hence log-divergent (behavior that is cured by the RI/MOM 
subtraction). On the other hand, the $x$ integral is convergent at $x=1$. Here the $\exp \left(-i x p^{z} z\right)$ term gives a "real" contribution with support in $-\infty<x<\infty$, while the $\exp \left(-i p^{z} z\right)$ gives a "virtual" contribution proportional to $\delta(1-x)$, and together they provide a well defined result at $x=1$. The one-loop correction to the local vector current is exactly zero as the above integral vanishes at $z=0$.

By imposing the condition in Eq. (2.1), we obtain the counterterm

$$
\tilde{q}_{\mathrm{CT}}^{(1)}\left(z, p_{R}^{z}, \mu_{R}\right)=-\left.\tilde{q}^{(1)}\left(z, p_{R}^{z}, 0, p^{2}\right)\right|_{p^{2}=-\mu_{R}^{2}} .
$$

To simplify the presentation of various formulae below we define the dimensionless ratio

$$
r_{R} \equiv \frac{\mu_{R}^{2}}{p_{R}^{z^{2}}} .
$$

In Euclidean space, $p_{E}^{2}=p_{4}^{2}+p_{R}^{z 2} \geq p_{z}^{2}$, so the renormalization scale $\mu_{R}$ one can reach on the lattice by setting $p_{E}^{2}=\mu_{R}^{2}$ always satisfies $\mu_{R}^{2} \geq p_{R}^{z 2}$. Therefore, we can consider $\tilde{q}_{\mathrm{CT}}^{(1)}$ after analytically continuing to the region $r_{R}>1$, which is easy to accomplish using the $i \varepsilon$ in Eq. (3.3).

Together these results give the renormalized one-loop quasi-PDF in the RI/MOM scheme [38]

$$
\tilde{q}_{\mathrm{OM}}^{(1)}\left(z, p^{z}, p_{R}^{z}, \mu_{R}\right)=\tilde{q}^{(1)}\left(z, p^{z}, 0, p^{2} \ll p^{z 2}\right)+\tilde{q}_{\mathrm{CT}}^{(1)}\left(z, p_{R}^{z}, \mu_{R}\right) .
$$

As indicated, to setup the matching of the quasi-PDF to the PDF, we must keep our physical IR regulator $p^{2}$ small, i.e. $\rho \ll 1$. Thus we identify the logarithmic IR divergences by Taylor expanding $\tilde{q}^{(1)}$ in $\rho$. At one-loop order there will only be a $\ln \rho$ term, corresponding to the leading logarithmic IR singularity which will also appear in the PDF. Also, our notation in Eq. (3.6) makes clear that the momentum $p^{z}$ of the state for which we are considering the quasi-PDF in general need not be equal to the momentum $p_{R}^{z}$ that we use for the RI/MOM counterterm.

The renormalized quasi-PDF in the RI/MOM scheme is therefore [38]

$$
\begin{aligned}
& \tilde{q}_{\mathrm{OM}}^{(1)}\left(z, p^{z}, p_{R}^{z}, \mu_{R}\right)=\frac{\alpha_{s} C_{F}}{2 \pi}\left(4 p^{z} \zeta\right) \int_{-\infty}^{\infty} d x\left(e^{-i x p^{z} z}-e^{-i p^{z} z}\right) \\
& \times \begin{cases}\frac{1+x^{2}}{1-x} \ln \frac{x}{x-1}-\frac{2}{\sqrt{r_{R}-1}}\left[\frac{1+x^{2}}{1-x}-\frac{r_{R}}{2(1-x)}\right] \arctan \frac{\sqrt{r_{R}-1}}{2 x-1}+\frac{r_{R}}{4 x(x-1)+r_{R}}, & x>1 \\
\frac{1+x^{2}}{1-x} \ln \frac{4\left(p^{z}\right)^{2}}{-p^{2}}-\frac{2}{\sqrt{r_{R}-1}}\left[\frac{1+x^{2}}{1-x}-\frac{r_{R}}{2(1-x)}\right] \arctan \sqrt{r_{R}-1}, & 0<x<1, \\
\frac{1+x^{2}}{1-x} \ln \frac{x-1}{x}+\frac{2}{\sqrt{r_{R}-1}}\left[\frac{1+x^{2}}{1-x}-\frac{r_{R}}{2(1-x)}\right] \arctan \frac{\sqrt{r_{R}-1}}{2 x-1}-\frac{r_{R}}{4 x(x-1)+r_{R}}, & x<0\end{cases}
\end{aligned}
$$

and the coupling $\alpha_{s}=\alpha_{s}(\mu)$ is taken to be in the standard $\overline{\mathrm{MS}}$ scheme. Note that the collinear divergence $\ln \rho$ only appears in the physical region of the PDF $0<x<1$. For $x \rightarrow \pm \infty$ the integrand for the renormalized quasi-PDF in Eq. (3.7) now behaves as $\propto 1 / x^{2}$ and hence the integral converges.

For the momentum space one-loop quasi-PDF $\tilde{q}_{\mathrm{OM}}^{(1)}\left(x, p^{z}, \mu_{R}\right)$ the difference of exponentials in Eq. (3.7) cause the functions of $x$ to act like plus-functions in each of the regions. To see this note that for any position space test function $g\left(z p^{z}\right)$ that

$$
\int \frac{d z}{2 \pi} g\left(z p^{z}\right)\left(e^{-i x p^{z} z}-e^{-i p^{z} z}\right)=[g(x)-g(1)],
$$


where $g(x)$ is the momentum space test function. We therefore define the following plus functions with subtractions at $x=1$

$$
\begin{aligned}
\int_{1}^{\infty} d x[h(x)]_{\oplus} g(x) & =\int_{1}^{\infty} d x h(x)[g(x)-g(1)], \\
\int_{0}^{1} d x\left(\frac{h(x)}{1-x}\right)_{+} g(x) & =\int_{0}^{1} d x \frac{h(x)}{1-x}[g(x)-g(1)], \\
\int_{-\infty}^{0} d x[h(x)]_{\ominus} g(x) & =\int_{-\infty}^{0} d x h(x)[g(x)-g(1)],
\end{aligned}
$$

for arbitrary functions $h(x)$ and $g(x)$. The renormalized momentum space quasi-PDF in the RI/MOM scheme in Feynman gauge is therefore [38]

$$
\begin{aligned}
& \tilde{q}_{\mathrm{OM}}^{(1)}\left(x, p^{z}, p_{R}^{z}, \mu_{R}\right) \\
& =\frac{\alpha_{s} C_{F}}{2 \pi}(4 \zeta) \\
& \times \begin{cases}{\left[\frac{1+x^{2}}{1-x} \ln \frac{x}{x-1}-\frac{2}{\sqrt{r_{R}-1}}\left[\frac{1+x^{2}}{1-x}-\frac{r_{R}}{2(1-x)}\right] \arctan \frac{\sqrt{r_{R}-1}}{2 x-1}+\frac{r_{R}}{4 x(x-1)+r_{R}}\right]_{\oplus}} & x>1 \\
{\left[\frac{1+x^{2}}{1-x} \ln \frac{4\left(p^{z}\right)^{2}}{-p^{2}}-\frac{2}{\sqrt{r_{R}-1}}\left[\frac{1+x^{2}}{1-x}-\frac{r_{R}}{2(1-x)}\right] \arctan \sqrt{r_{R}-1}\right]_{+}} & 0<x<1 . \\
{\left[\frac{1+x^{2}}{1-x} \ln \frac{x-1}{x}+\frac{2}{\sqrt{r_{R}-1}}\left[\frac{1+x^{2}}{1-x}-\frac{r_{R}}{2(1-x)}\right] \arctan \frac{\sqrt{r_{R}-1}}{2 x-1}-\frac{r_{R}}{4 x(x-1)+r_{R}}\right]_{\ominus}} & x<0\end{cases}
\end{aligned}
$$

Next we consider the PDF calculated with the same off-shellness regulator, once again using the same identity in Eq. (2.4) to uniquely define our treatment of the spinors when working off-shell. With this definition the renormalized one-loop matrix element of PDF in the $\overline{\mathrm{MS}}$ scheme is given by

$$
q^{(1)}(x, \mu)=\frac{\alpha_{s} C_{F}}{2 \pi}(4 \zeta)\left\{\begin{array}{cc}
0 & x>1 \\
{\left[\frac{1+x^{2}}{1-x} \ln \frac{\mu^{2}}{-p^{2}}-\frac{1+x^{2}}{1-x} \ln [x(1-x)]-(2-x)\right]_{+}} & 0<x<1 . \\
0 & x<0
\end{array} .\right.
$$

Considering the factorization formula in Eq. (2.3), the matching coefficient $C^{\mathrm{OM}}$ between the renormalized quasi-PDF in the RI/MOM scheme and standard PDF in the $\overline{\mathrm{MS}}$ scheme is then determined by the difference between the momentum space quasi-PDF and PDF results

$$
C^{\mathrm{OM}}\left(\xi, \frac{\mu_{R}}{p_{R}^{z}}, \frac{\mu}{p^{z}}\right)=\delta(1-\xi)+\frac{1}{4 \zeta}\left[\tilde{q}_{\mathrm{OM}}^{(1)}\left(\xi, p^{z}, p_{R}^{z}, \mu_{R}\right)-q^{(1)}(\xi, \mu)\right]+\mathscr{O}\left(\alpha_{s}^{2}\right),
$$


which gives the one-loop matching coefficient [38]

$$
\begin{aligned}
& C^{\mathrm{OM}}\left(\xi, \frac{\mu_{R}}{p_{R}^{z}}, \frac{\mu}{p^{z}}\right) \\
= & \delta(1-\xi)+\frac{\alpha_{s} C_{F}}{2 \pi} \\
\times & \begin{cases}{\left[\frac{1+\xi^{2}}{1-\xi} \ln \frac{\xi}{\xi-1}-\frac{2\left(1+\xi^{2}\right)-r_{R}}{(1-\xi) \sqrt{r_{R}-1}} \arctan \frac{\sqrt{r_{R}-1}}{2 \xi-1}+\frac{r_{R}}{4 \xi(\xi-1)+r_{R}}\right]_{\oplus}} & \xi>1 \\
{\left[\frac{1+\xi^{2}}{1-\xi} \ln \frac{4\left(p^{z}\right)^{2}}{\mu^{2}}+\frac{1+\xi^{2}}{1-\xi} \ln [\xi(1-\xi)]+(2-\xi)-\frac{2 \arctan \sqrt{r_{R}-1}}{\sqrt{r_{R}-1}} \frac{1+\xi^{2}-r_{R} / 2}{(1-\xi)}\right]_{+}} & 0<\xi<1, \\
{\left[\frac{1+\xi^{2}}{1-\xi} \ln \frac{\xi-1}{\xi}+\frac{2}{\sqrt{r_{R}-1}}\left[\frac{1+\xi^{2}}{1-\xi}-\frac{r_{R}}{2(1-\xi)}\right] \arctan \frac{\sqrt{r_{R}-1}}{2 \xi-1}-\frac{r_{R}}{4 \xi(\xi-1)+r_{R}}\right]_{\ominus} \quad \xi<0} & \end{cases}
\end{aligned}
$$

where $r_{R}$ is given in Eq. (3.5). This is the result for the matching between quasi-PDF in the $\mathrm{RI} / \mathrm{MOM}$ scheme and PDF in the $\overline{\mathrm{MS}}$ scheme for the non-singlet case in Feynman gauge. As expected, $C_{\mathrm{OM}}$ is independent of the IR regulator $-p^{2}$ since the logarithmic IR singularities cancel between the quasi-PDF and PDF.

The result in the RI/MOM scheme in Eq. (3.13) also exhibits convergent behavior as $\xi \rightarrow \pm \infty$. In section 3.2 below we compare this behavior with the results obtained with the quasi-PDF in the $\overline{\mathrm{MS}}$ and transverse cutoff schemes.

\subsection{Landau and General Covariant Gauge}

In lattice QCD, the RI/MOM scheme is most easily implemented in the Landau gauge, so in this section we extend our calculation to give the matching result for a general covariant gauge where the gluon propagator is

$$
i D_{\tau}^{\mu v}(k)=-\frac{i}{k^{2}}\left[g^{\mu v}-(1-\tau) \frac{k^{\mu} k^{v}}{k^{2}}\right] .
$$

Here $\tau=0$ corresponds to the Landau gauge. We write the quasi-PDF in a general covariant gauge as the $\tau=1$ Feynman gauge result plus a correction

$$
\tilde{q}_{\tau}^{\mathrm{OM}}\left(x, p^{z}, p_{R}^{z}, \mu_{R}\right)=\tilde{q}^{\mathrm{OM}}\left(x, p^{z}, p_{R}^{z}, \mu_{R}\right)+\Delta \tilde{q}_{\tau}^{\mathrm{OM}}\left(x, p^{z}, p_{R}^{z}, \mu_{R}\right) .
$$

The correction from the $(1-\tau)$ term in Eq. (3.14) is at one-loop given by [38]

$$
\Delta \tilde{q}_{\tau}^{\mathrm{OM}}\left(x, \rho, r_{R}\right)=(1-\tau) \frac{\alpha_{s} C_{F}}{2 \pi}(4 \zeta)\left\{\begin{array}{lc}
\left(\frac{(1-2 x) r_{R}^{2}}{2(1-x)\left[r_{R}+4 x(x-1)\right]^{2}}\right)_{\oplus} & x>1 \\
0 & 0<x<1 \\
\left(\frac{-(1-2 x) r_{R}^{2}}{2(1-x)\left[r_{R}+4 x(x-1)\right]^{2}}\right)_{\ominus} & x<0
\end{array}\right.
$$


For the PDF with an off-shell momentum regulator, there is also an additional contribution,

$$
q_{\tau}^{(1)}(x, \mu)=(1-\tau) \frac{\alpha_{s} C_{F}}{2 \pi}(4 \zeta)\left\{\begin{array}{lc}
0 & x>1 \\
\left(\frac{1-2 x}{2(1-x)}\right)+ & 0<x<1 . \\
0 & x<0
\end{array} .\right.
$$

As a result, the matching coefficient for a general covariant gauge is given by the Feynman gauge result from Eq. (3.13) plus an additional term [38]

$C_{\tau}^{\mathrm{OM}}\left(\xi, \frac{\mu_{R}}{p_{R}^{z}}, \frac{\mu}{p^{z}}\right)=C^{\mathrm{OM}}\left(\xi, \frac{\mu_{R}}{p_{R}^{z}}, \frac{\mu}{p^{z}}\right)+(1-\tau) \frac{\alpha_{s} C_{F}}{2 \pi} \begin{cases}\left(\frac{(1-2 \xi)}{2(1-\xi)} \frac{r_{R}^{2}}{\left[r_{R}+4 \xi(\xi-1)\right]^{2}}\right)_{\oplus} & \xi>1 \\ \left(-\frac{(1-2 \xi)}{2(1-\xi)}\right)_{+} & 0<\xi<1 . \\ \left(-\frac{(1-2 \xi)}{2(1-\xi)} \frac{r_{R}^{2}}{\left[r_{R}+4 \xi(\xi-1)\right]^{2}}\right)_{\ominus} & \xi<0\end{cases}$

When utilizing the matching equation with the RI/MOM scheme in a chosen gauge, we note that the RI/MOM quasi-PDF $\tilde{q}_{\tau}^{\mathrm{OM}}$ is gauge dependent, as is the matching coefficient $C_{\tau}^{\mathrm{OM}}$, and in both cases this is induced by the presence of the gauge dependent RI/MOM UV counterterm. Therefore this gauge dependence is the same and yields a gauge invariant result for the $\overline{\mathrm{MS}} \mathrm{PDF}$ order by order in $\alpha_{s}$. When the quasi-PDF is renormalized non-perturbatively and the matching is carried out perturbatively, then the cancellation will be incomplete, and it would be reasonable for example to look at the residual gauge dependence as a means of assessing an uncertainty from higher orders in perturbation theory. However we will see in Sec. 4 that at one-loop the gauge dependent terms are much smaller than the residual scale dependence, and hence this is unlikely to be a significant source of uncertainty. For our numerical analysis in Sec. 4 we will consider both the Feynman gauge result from Eq. (3.13), denoted $C^{\mathrm{OM}}$, and the Landau gauge result obtained from Eq. (3.17) with $\tau=0$, and denoted $C_{\tau=0}^{\mathrm{OM}}$.

\subsection{Comparison to Other Schemes}

The one-loop matching coefficient between the quasi-PDF and PDF was originally calculated in Ref. [25] in an on-shell scheme with the UV divergence regulated by a finite transverse momentum cutoff $\Lambda_{T}$, using Feynman gauge. Using our notation for the plus functions the result for this scheme is [25] 


$$
\begin{aligned}
& C^{\Lambda_{T}}\left(\xi, \frac{\mu}{p^{z}}, \frac{\Lambda}{P^{z}}\right) \\
& =\delta(1-\xi) \\
& +\frac{\alpha_{s} C_{F}}{2 \pi}\left\{\begin{array}{cl}
{\left[\frac{1+\xi^{2}}{1-\xi} \ln \frac{\xi}{\xi-1}+1+\frac{1}{(1-\xi)^{2}} \frac{\Lambda_{T}}{P^{z}}\right]_{\oplus}} & \xi>1 \\
{\left[\frac{1+\xi^{2}}{1-\xi} \ln \frac{4\left(p^{z}\right)^{2}}{\mu^{2}}+\frac{1+\xi^{2}}{1-\xi} \ln \xi(1-\xi)+1-\frac{2 \xi}{1-\xi}+\frac{1}{(1-\xi)^{2}} \frac{\Lambda_{T}}{P^{z}}\right]_{+}} & 0<\xi<1 . \\
{\left[\frac{1+\xi^{2}}{1-\xi} \ln \frac{\xi-1}{\xi}-1+\frac{1}{(1-\xi)^{2}} \frac{\Lambda_{T}}{P^{z}}\right]_{\ominus}} & \xi<0
\end{array}\right.
\end{aligned}
$$

This result was used in the lattice calculations of $f_{u-d}$ in Refs. $[17,18,19,20]$. Note that the linear divergence is not subtracted in the quasi-PDF in this scheme, so there is no renormalization scale $\mu_{R}$ associated with it.

In the recent works [30, 22], the quasi-PDF is renormalized in the RI/MOM scheme and matched to the quasi-PDF in the $\overline{\mathrm{MS}}$ scheme. Eventually, the quasi-PDF in the $\overline{\mathrm{MS}}$ scheme needs to be matched to PDF in the $\overline{\mathrm{MS}}$ scheme. In this case, the matching coefficient is very similar to that in Ref. [25] except for the vanishing of the linear divergence, and a different polynomial $\xi$ dependence in the $0<\xi<1$ region [38],

$$
\begin{aligned}
& C^{\overline{\mathrm{MS}}}\left(\xi, \frac{\mu}{p^{z}}\right) \\
& =\delta(1-\xi)+\frac{\alpha_{s} C_{F}}{2 \pi}\left\{\begin{array}{cc}
{\left[\frac{1+\xi^{2}}{1-\xi} \ln \frac{\xi}{\xi-1}+1\right]_{\oplus}} & \xi>1 \\
{\left[\frac{1+\xi^{2}}{1-\xi} \ln \frac{4\left(p^{z}\right)^{2}}{\mu^{2}}+\frac{1+\xi^{2}}{1-\xi} \ln \xi(1-\xi)+(2-\xi)-\frac{2 \xi}{1-\xi}\right]_{+}} & 0<\xi<1 . \\
{\left[\frac{1+\xi^{2}}{1-\xi} \ln \frac{\xi-1}{\xi}-1\right]_{\ominus}} & \xi<0
\end{array}\right.
\end{aligned}
$$

This result for $C^{\overline{\mathrm{MS}}}$ is gauge invariant, which follows because on-shell definitions of the quasiPDF and PDF in the $\overline{\mathrm{MS}}$ scheme are gauge invariant (or alternatively because any gauge dependence associated with off-shell regulation of the infrared physics will be identical for the quasi-PDF and PDF).

\section{Numerical Analysis}

In this section we numerically analyze the quasi-PDF by studying how the matching coefficient in Eq. (1.8) changes the PDF. To calculate the convolution between the momentum space matching coefficient $C^{\mathrm{OM}}(x / y)$ and a PDF $f(y)$ we note that for $-1<y<1$ and fixed $x$ the variable $x / y$ goes over the range $-\infty<x / y<-|x|$ and $|x|<x / y<\infty$, and we can simply implement the integration 

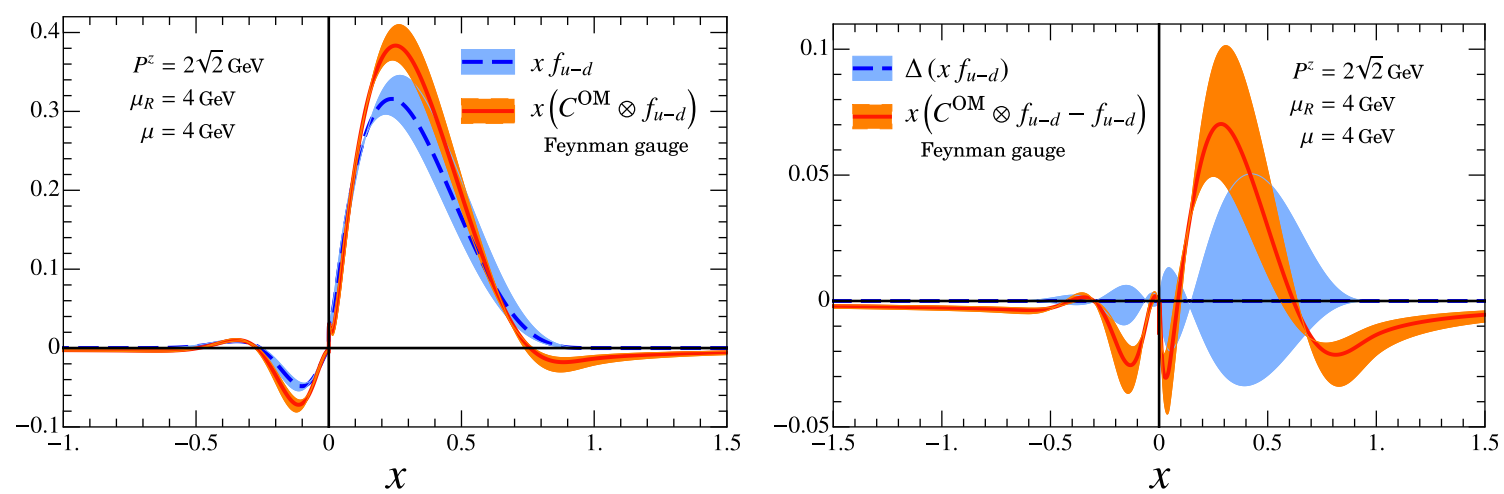

Figure 2: Comparison between the PDF $x f_{u-d}$ and the quasi-PDF result obtained from $x\left(C^{\mathrm{OM}} \otimes f_{u-d}\right)$ in Feynman gauge. The orange and blue bands indicate the results from varying the factorization scale $\mu$ by a factor of two. Left: $x\left(C^{\mathrm{OM}} \otimes f_{u-d}\right)$ and $x f_{u-d}$. Right: differences between $x\left(C^{\mathrm{OM}} \otimes f_{u-d}\right), x f_{u-d}$, and $x f_{u-d}(x, 4 \mathrm{GeV})$.

using the plus functions in Eq. (3.9). Suppressing the $\mu / p^{z}$ and $\mu_{R} / p_{R}^{z}$ arguments, and absorbing the limits $\theta(1-|y|)$ in the function $f(y)$ this gives

$$
\begin{aligned}
\int d y C^{\mathrm{OM}}\left(\frac{x}{y}\right) \frac{f(y)}{|y|} & =\int d y^{\prime} C_{r}^{\mathrm{OM}}\left(y^{\prime}\right)\left[\frac{x}{y^{\prime 2}} \frac{f\left(x / y^{\prime}\right)}{\left|x / y^{\prime}\right|}-x \frac{f(x)}{|x|}\right] \\
& =\int_{-\infty}^{\infty} d y\left[\frac{1}{|y|} C_{r}^{\mathrm{OM}}\left(\frac{x}{y}\right) f(y)-\frac{1}{|x|} C_{r}^{\mathrm{OM}}\left(\frac{y}{x}\right) f(x)\right] .
\end{aligned}
$$

Here the subscript $r$ on $C_{r}^{\mathrm{OM}}$ denotes the pure function, which are the argument of the plus functions. For the first equality in Eq. (4.1) we changed variable to $y^{\prime}=x / y$ and then applied the plus functions. For the last equality in Eq. (4.1) we changed variable back to $y=x / y^{\prime}$ in the first term, and to $y^{\prime}=y / x$ in the second term.

As an example we use for our analysis the unpolarized iso-vector parton distribution,

$$
f_{u-d}(x, \mu)=f_{u}(x, \mu)-f_{d}(x, \mu)-f_{\bar{u}}(-x, \mu)+f_{\bar{d}}(-x, \mu)
$$

where we include $f_{\bar{u}}(-x, \mu)=-f_{\bar{u}}(x, \mu)$ and $f_{\bar{d}}(-x, \mu)=-f_{\bar{d}}(x, \mu)$, the anti-parton distributions. We use the next-to-leading-order iso-vector PDF $f_{u-d}$ from "MSTW 2008" [3] with the corresponding running coupling $\alpha_{s}(\mu)$. For the numerical calculation we impose a UV cutoff on the $y$-integral so that $|y|<y_{\text {cut }}=10^{n}$ for any $x$ and some $n>1$. Results in the RI/MOM scheme are independent of this cutoff, whereas we will show below that the transverse cutoff and $\overline{\mathrm{MS}}$ schemes exhibit sensitivity to $y_{\text {cut }}$. We also test soft cutoffs $|y|>10^{-m}$ and $|y-x|>10^{-m}$ with $m>3$, but find that the results in all schemes are independent of this $m$.

As default values for our figures we take $P^{z}=2 \sqrt{2} \mathrm{GeV}$, use the Feynman gauge RI/MOM matching result $C^{\mathrm{OM}}$ from Eq. (3.13) and the $\overline{\mathrm{MS}}$ renormalization scale $\mu=4 \mathrm{GeV}$. To specify the RI/MOM scheme parameters we take the renormalization scale $\mu_{R}=4 \mathrm{GeV}$ and momentum $p_{R}^{z}=P^{z}$. We consider variations about each of these default choices.

First consider Fig. 2 which shows a comparison of the RI/MOM quasi-PDF $\tilde{q}^{\mathrm{OM}}\left(x, P^{z}, p_{R}^{z}, \mu_{R}\right)=$ $\left(C^{\mathrm{OM}} \otimes f_{u-d}\right)$ (red solid line) and the $\overline{\mathrm{MS}} \mathrm{PDF} f_{u-d}(x, \mu)$ (blue dashed line). In this figure and in 

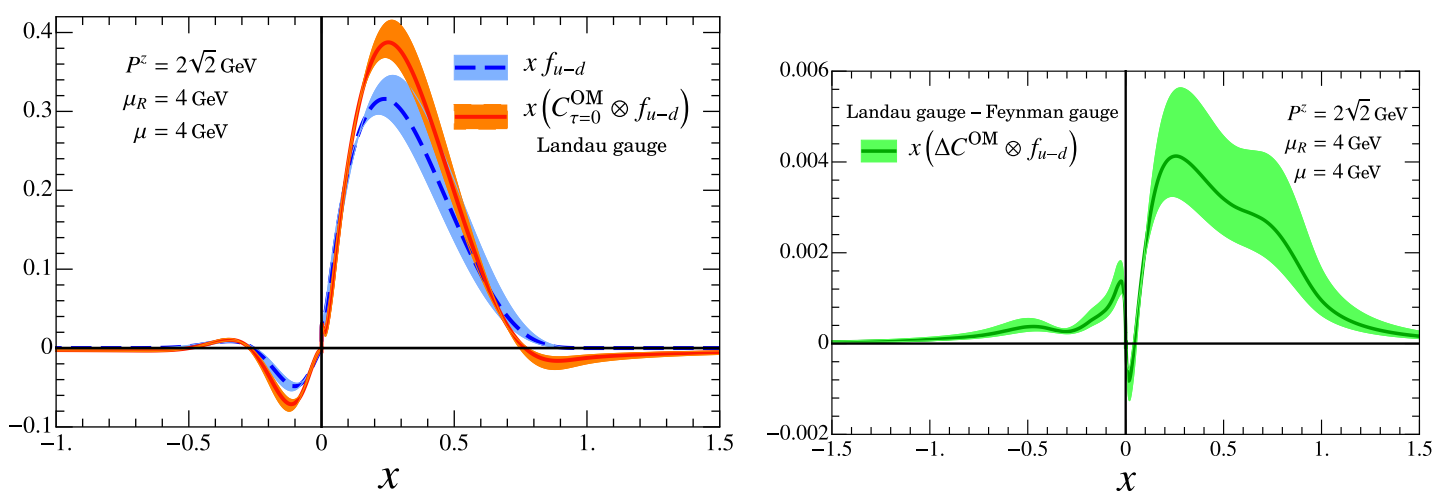

Figure 3: Comparison between the PDF $x f_{u-d}$ and the quasi-PDF obtained from $x\left(C^{\mathrm{OM}} \otimes f_{u-d}\right)$ in the Landau gauge. The orange, blue, and green bands indicate the results from varying the factorization scale $\mu$ by a factor of two.
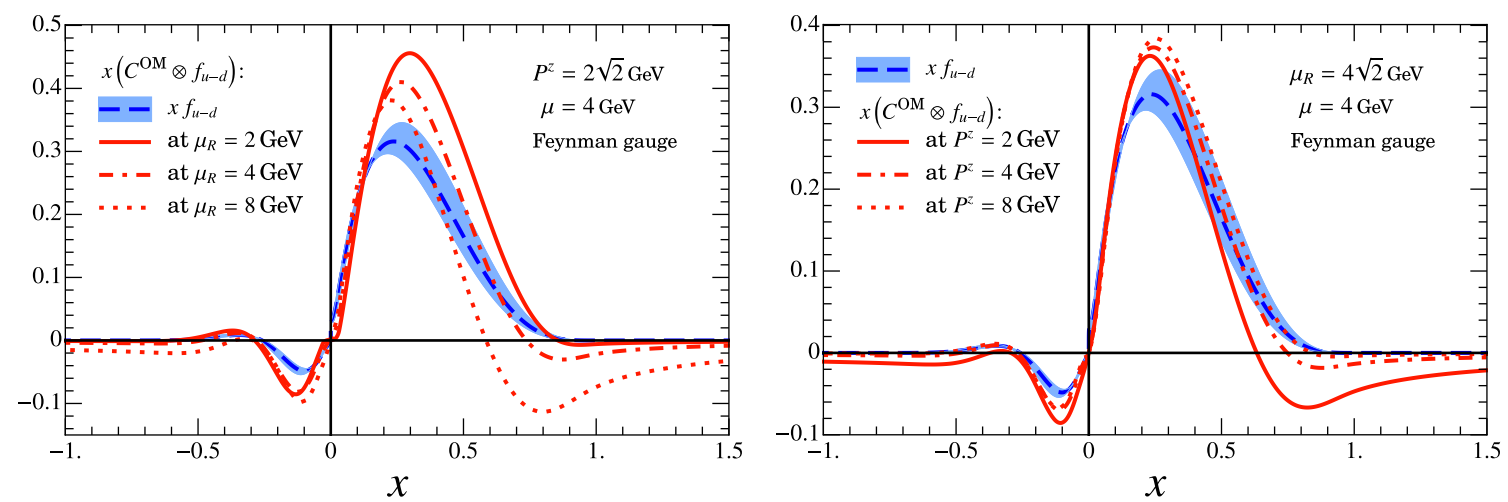

Figure 4: Left panel: Comparison between the PDF $x f_{u-d}$ and the quasi-PDF from $x\left(C^{\mathrm{OM}} \otimes f_{u-d}\right)$ determined at different $\mu_{R}$ s. Right panel: The $P^{z}$ dependence of the quasi-PDF $x\left(C^{\mathrm{OM}} \otimes f_{u-d}\right)$, compared to the PDF $x f_{u-d}$ which is independent of $P^{z}$. In both panels the blue band indicates the $\mu$ renormalization scale dependence of the PDF from variation by a factor of two.

others below we multiply by $x$ in order to more easily observe the small $x$ region. The left panel shows the direct comparison, and the right panel makes the comparison subtracting $x f_{u-d}(x, \mu)$. We see that the quasi-PDF and PDF are close to one another, which is appealing for the convergence of perturbation theory. In Fig. 2 we also vary the factorization scale $\mu$ by a factor of two, from $\mu=2 \mathrm{GeV}$ to $\mu=8 \mathrm{GeV}$, showing the result by the blue and orange bands about the PDF and quasi-PDF respectively. For the quasi-PDF from Eq. (3.13) the dependence on $\mu$ cancels out between $C$ and $q$, order by order in perturbation theory, whereas the PDF has a dependence on $\mu$ at leading-logarithmic order, and this decrease in the $\mu$ dependence is observed as expected. As shown in Fig. 2, the $C^{\mathrm{OM}} \otimes f_{u-d}$ has small non-zero values outside the region $-1<x<1$. To examine these differences more closely, we subtract the central curve $x f_{u-d}(x, \mu=4 \mathrm{GeV})$ from both $x f_{u-d}(x, \mu)$ and $x\left(C^{\mathrm{OM}} \otimes f_{u-d}\right)$, and plot their differences in the right panel of Fig. 2.

Next we examine the gauge dependence of the quasi-PDF in the RI/MOM scheme. The result for Landau gauge ( $\tau=0$ ) is shown in the left panel of Fig. 3, which is plotted in the same way as 
Fig. 2, and appears very similar. To examine the change to the quasi-PDF we therefore plot the difference between the Landau gauge and Feynman gauge result in the right panel of Fig. 3. We see that the the $\tau$-dependent contribution in the matching coefficient are very small, on average being only a $1 \%$ correction. Thus switching gauge only results in a small change to the matching coefficient.

Our next step is to fix the factorization scale at $\mu=4 \mathrm{GeV}$ and vary the renormalization scale $\mu_{R}$ in the quasi-PDF. From Eq. (3.10) we see that the definition of the RI/MOM quasi-PDF depends on the scale parameter $\mu_{R}$, so different $\mu_{R}$ s correspond to different quasi-PDfs. For $\mu_{R}=4,8,16$ $\mathrm{GeV}$, we plot in the left panel of Fig. 4 a comparison between $x\left(C^{\mathrm{OM}} \otimes f_{u-d}\right)\left(x, \mu_{R}\right)$ and $x f_{u-d}(x, \mu)$ with $\mu=4 \mathrm{GeV}$ (blue dashed line). The blue band shows how the PDF changes when we take $\mu=2$ or $8 \mathrm{GeV}$. We see that the RI/MOM quasi-PDF is quite sensitive to the choice of $\mu_{R}$, exhibiting larger variations than that of varying the renormalization scale $\mu$ in the PDF. We also observe that the quasi-PDF moves away from the PDF when $\mu_{R}>\mu$.

In the right panel of Fig. 4 we hold $\mu_{R}=4 \sqrt{2} \mathrm{GeV}$ fixed, and vary $P^{z}$. We observe that in the tails $(x>1$ and $x<-1)$ that the RI/MOM quasi-PDF approaches the PDF in the anticipated power-law fashion as $P^{z}$ gets large. On the other hand, in the central region $-1<x<1$ the matching coefficient becomes a finite function in the $P^{z} \rightarrow \infty$ limit, and hence there is always perturbative conversion needed between the quasi-PDF and PDF.

Finally we consider the comparison between our RI/MOM results and the matching results in the transverse cut-off and $\overline{\mathrm{MS}}$ schemes. At $P^{z}=2 \sqrt{2} \mathrm{GeV}, \mu=\mu_{R}=4 \mathrm{GeV}$, and $\Lambda_{\mathrm{T}}=4 \mathrm{GeV}$, we calculate $C^{\Lambda_{\mathrm{T}}} \otimes f_{u-d}$ and $C^{\overline{\mathrm{MS}}} \otimes f_{u-d}$ with $y_{\text {cut }}=10^{1}, 10^{2}, 10^{4}$ and plot the results with comparison to $f_{u-d}$ in Fig. 5. Unlike the $C^{\mathrm{OM}} \otimes f_{u-d}$, both $C^{\Lambda_{\mathrm{T}}} \otimes f_{u-d}$ and $C^{\overline{\mathrm{MS}}} \otimes f_{u-d}$ suffer from UV divergences in the integration over $y$, and they differ significantly from $f_{u-d}$. This means that when one inverts the factorization formula in Eq. (1.8) to determine the PDF from the quasi-PDF, that there must be a large cancellation of UV divergences between the quasi-PDF from lattice QCD and the matching coefficient in the convolution integral. Since the UV region of a matching factor $C(x / y)$ is near $y=0$, it is necessary to test the sensitivity of the convolution integral to the smallest momentum fraction of the quasi-PDF for the lattice calculations in Ref. [17, 18, 19, 20, 22]. Using the RI/MOM scheme avoids this complication. Another advantage of the RI/MOM scheme is that in the unphysical regions $|x|>1$ the $\alpha_{s}$ corrections to the matching coefficient fall as $1 /\left(P^{z}\right)^{2}$ as $P^{z} \rightarrow \infty$, so the quasi-PDF will vanish in this region. This is not the case for the quasi-PDF in the transverse cutoff and $\overline{\mathrm{MS}}$ schemes, where the quasi-PDF asymptotes to a non-trivial function for $|x|>1$ when $P^{z} \rightarrow \infty$.

The reason why $C^{\mathrm{OM}} \otimes f_{u-d}$ has better UV convergence than the other two schemes is that only the RI/MOM scheme introduces a counterterm to the quasi-PDF which will cancel out the UV divergences that arises when one integrates over $y$. The matching result in the RI/MOM scheme therefore yields only a bounded small effect which one can be more confident about treating perturbatively. Thus, to reduce the uncertainties, our results imply that it is reasonable to favor RI/MOM over the other two schemes.

\section{5. conclusion}

We have described the procedure of nonperturbative renormalization of quasi-PDF in the 

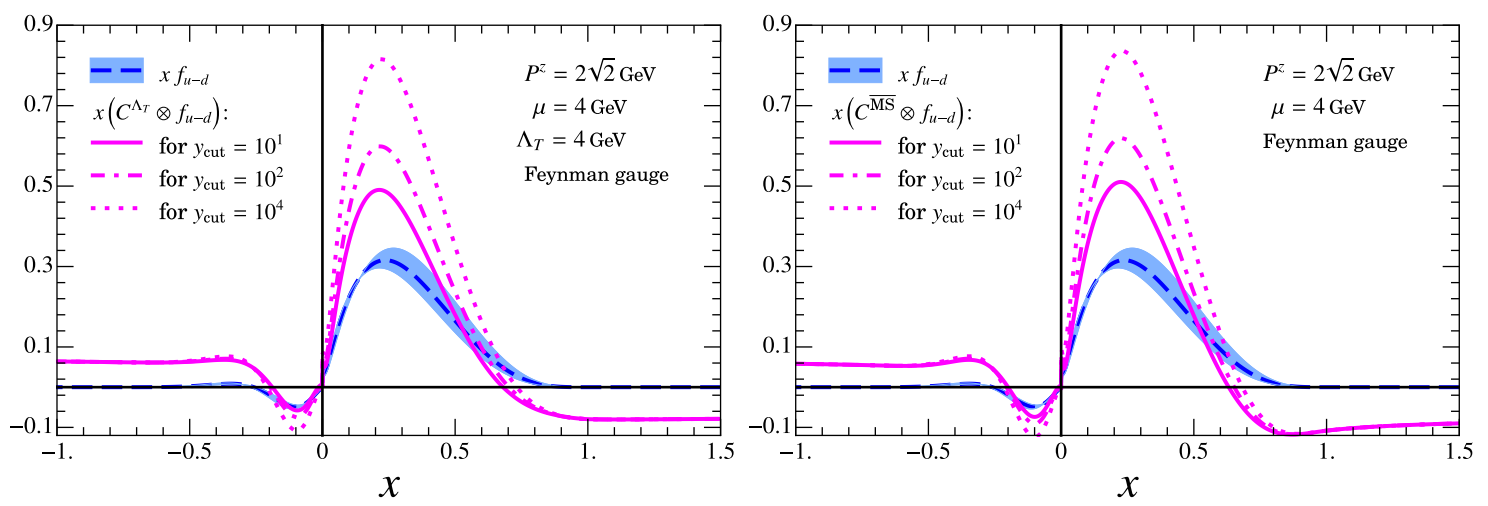

Figure 5: Results for the quasi-PDF in other schemes compared to the PDF $f_{u-d}$. The left panel shows $x\left(C^{\Lambda_{T}} \otimes f_{u-d}\right)$ with three different values for $y_{\text {cut }}$. The right panel shows $x\left(C^{\overline{\mathrm{MS}}} \otimes f_{u-d}\right)$ also with three different values for $y_{\text {cut }}$.

RI/MOM scheme. The $z$-dependent renormalization constant is obtained by imposing Eq. (2.1) on the off-shell quark matrix element of the spatial correlation operator in lattice QCD. Then the renormalization constant is applied to the nucleon matrix element of the same correlation operator whose Fourier transform gives the quasi-PDF on the lattice. In RI/MOM the renormalized quasi$\mathrm{PDF}$ is regularization invariant and can be related to PDF in the $\overline{\mathrm{MS}}$ scheme through a perturbative matching condition, which is calculable in the continuum theory with dimensional regularization. Since all the large corrections in lattice perturbation theory are absorbed into the nonperturbative renormalization constant, the uncertainty of this procedure comes from lattice discretization effects and perturbative matching in the continuum theory. Our numerical results show that the one-loop matching for the RI/MOM scheme has nice UV convergence and reasonable magnitude for a perturbative correction, which is in contrast to the matching results for the $\overline{\mathrm{MS}}$ or transverse-momentum cutoff schemes. This indicates that the theoretical uncertainty in the perturbative matching for the RI/MOM scheme is small and controllable, thus making it more favorable than the $\overline{\mathrm{MS}}$ and transverse-momentum cutoff schemes. Furthermore, the matching in the RI/MOM scheme is consistent with the quasi-PDF vanishing in the unphysical region $|x|>1$ as $P^{z} \rightarrow \infty$, unlike the results in the transverse cutoff or $\overline{\mathrm{MS}}$ schemes. To increase the accuracy of our results in the future, one can study the $O(a)$ improvement for the lattice simulation of quasi-PDF and calculate the matching coefficient to higher orders in perturbation theory. As the capabilities for doing simulations with larger nucleon momentum on the lattice continue to improve, we believe that our results will provide an important ingredient for future lattice calculations of PDF with the desired accuracy.

It should be noted that on the lattice there are not only discretization errors, but also mixings between the quasi-PDF and other operators due to the broken symmetries. We should include all the possible operators that mix with the gauge-invariant quark bilinear and determine their renormalization constants nonperturbatively [22, 30, 23, 31]. We must also be aware of the fact that one can only calculate the spatial correlation for the quasi-PDF at a finite number of discrete $z$ 's on the lattice. As a result, its Fourier transform into momentum space will exhibit an oscillatory behavior due to the truncation at $\left|z_{\max }\right|[22,23,31]$. Nevertheless, if we derive the parametric behavior of the spatial correlation at large $|z|$, we can use a proper set of basis functions to fit the 
data points and obtain a smooth curve. Then we can Fourier transform this smooth curve to obtain the quasi-PDF, which is free of the truncation error and should capture the correct behavior in the small $x$ region that can only be probed at large $|z|$. The choice of the basis functions could be based on results from global fits to the PDF with matching correction in Eq. (1.8). Two other approaches to reduce the truncation error were also proposed in the recent paper [24].

\section{Acknowledgement}

The authors are thankful for discussions with J. W. Chen, W. Detmold, M. Constantinou, L. Jin, Y. B. Yang, K. F. Liu, J. H. Zhang, M. Engelhardt, P. Shanahan and D. Horkel. This material was supported by the U.S. Department of Energy, Office of Science, Office of Nuclear Physics, from DE-SC0011090 and within the framework of the TMD Topical Collaboration. I.S. was also supported in part by the Simons Foundation through the Investigator grant 327942.

\section{References}

[1] R. D. Ball et al. [NNPDF Collaboration], "Parton distributions for the LHC Run II," JHEP 1504, 040 (2015) doi:10.1007/JHEP04(2015)040 [arXiv:1410.8849 [hep-ph]].

[2] S. Dulat et al., "New parton distribution functions from a global analysis of quantum chromodynamics," Phys. Rev. D 93, no. 3, 033006 (2016) doi:10.1103/PhysRevD.93.033006 [arXiv:1506.07443 [hep-ph]].

[3] A. D. Martin, W. J. Stirling, R. S. Thorne and G. Watt, "Parton distributions for the LHC," Eur. Phys. J. C 63, 189 (2009) doi:10.1140/epjc/s10052-009-1072-5 [arXiv:0901.0002 [hep-ph]].

[4] S. Alekhin, J. Blümlein, S. Moch and R. Placakyte, "Parton distribution functions, $\alpha_{s}$, and heavy-quark masses for LHC Run II," Phys. Rev. D 96, no. 1, 014011 (2017) doi:10.1103/PhysRevD.96.014011 [arXiv:1701.05838 [hep-ph]].

[5] A. Buckley, J. Ferrando, S. Lloyd, K. Nordström, B. Page, M. Rüfenacht, M. Schönherr and G. Watt, "LHAPDF6: parton density access in the LHC precision era," Eur. Phys. J. C 75, 132 (2015) doi:10.1140/epjc/s10052-015-3318-8 [arXiv:1412.7420 [hep-ph]].

[6] W. Detmold, W. Melnitchouk and A. W. Thomas, "Parton distributions from lattice QCD," Eur. Phys. J. direct 3, no. 1, 13 (2001) doi:10.1007/s1010501c0013 [hep-lat/0108002].

[7] W. Detmold, W. Melnitchouk and A. W. Thomas, "Moments of isovector quark distributions from lattice QCD,” Phys. Rev. D 66, 054501 (2002) doi:10.1103/PhysRevD.66.054501 [hep-lat/0206001].

[8] D. Dolgov et al. [LHPC and TXL Collaborations], "Moments of nucleon light cone quark distributions calculated in full lattice QCD," Phys. Rev. D 66, 034506 (2002) doi:10.1103/PhysRevD.66.034506 [hep-lat/0201021].

[9] X. Ji, "Parton Physics on a Euclidean Lattice," Phys. Rev. Lett. 110, 262002 (2013) doi:10.1103/PhysRevLett.110.262002 [arXiv:1305.1539 [hep-ph]].

[10] I. W. Stewart, F. J. Tackmann and W. J. Waalewijn, "Factorization at the LHC: From PDFs to Initial State Jets," Phys. Rev. D 81, 094035 (2010) doi:10.1103/PhysRevD.81.094035 [arXiv:0910.0467 [hep-ph]].

[11] I. W. Stewart, F. J. Tackmann and W. J. Waalewijn, “The Quark Beam Function at NNLL, JHEP 1009, 005 (2010) doi:10.1007/JHEP09(2010)005 [arXiv:1002.2213 [hep-ph]]. 
[12] X. Ji and J. H. Zhang, "Renormalization of quasiparton distribution,” Phys. Rev. D 92, 034006 (2015) doi:10.1103/PhysRevD.92.034006 [arXiv:1505.07699 [hep-ph]].

[13] X. Ji, J. H. Zhang and Y. Zhao, "Renormalization in Large Momentum Effective Theory of Parton Physics," arXiv:1706.08962 [hep-ph].

[14] T. Ishikawa, Y. Q. Ma, J. W. Qiu and S. Yoshida, “On the Renormalizability of Quasi Parton Distribution Functions,” arXiv:1707.03107 [hep-ph].

[15] X. Ji, "Parton Physics from Large-Momentum Effective Field Theory," Sci. China Phys. Mech. Astron. 57, 1407 (2014) doi:10.1007/s11433-014-5492-3 [arXiv:1404.6680 [hep-ph]].

[16] T. Izubuchi, X. Ji, L. Jin, I. Stewart and Y. Zhao, "Factorization Theorem Relating Euclidean and Light-Cone Parton Distributions," to bu published.

[17] H. W. Lin, J. W. Chen, S. D. Cohen and X. Ji, "Flavor Structure of the Nucleon Sea from Lattice QCD,” Phys. Rev. D 91, 054510 (2015) doi:10.1103/PhysRevD.91.054510 [arXiv:1402.1462 [hep-ph]].

[18] C. Alexandrou, K. Cichy, V. Drach, E. Garcia-Ramos, K. Hadjiyiannakou, K. Jansen, F. Steffens and C. Wiese, "Lattice calculation of parton distributions," Phys. Rev. D 92, 014502 (2015) doi:10.1103/PhysRevD.92.014502 [arXiv:1504.07455 [hep-lat]].

[19] J. W. Chen, S. D. Cohen, X. Ji, H. W. Lin and J. H. Zhang, "Nucleon Helicity and Transversity Parton Distributions from Lattice QCD,” Nucl. Phys. B 911, 246 (2016) doi:10.1016/j.nuclphysb.2016.07.033 [arXiv:1603.06664 [hep-ph]].

[20] C. Alexandrou, K. Cichy, M. Constantinou, K. Hadjiyiannakou, K. Jansen, F. Steffens and C. Wiese, “Updated Lattice Results for Parton Distributions," Phys. Rev. D 96, no. 1, 014513 (2017) doi:10.1103/PhysRevD.96.014513 [arXiv:1610.03689 [hep-lat]].

[21] J. H. Zhang, J. W. Chen, X. Ji, L. Jin and H. W. Lin, "Pion Distribution Amplitude from Lattice QCD,” Phys. Rev. D 95, no. 9, 094514 (2017) doi:10.1103/PhysRevD.95.094514 [arXiv:1702.00008 [hep-lat]].

[22] C. Alexandrou, K. Cichy, M. Constantinou, K. Hadjiyiannakou, K. Jansen, H. Panagopoulos and F. Steffens, "A complete non-perturbative renormalization prescription for quasi-PDFs," Nucl. Phys. B 923, 394 (2017) doi:10.1016/j.nuclphysb.2017.08.012 [arXiv:1706.00265 [hep-lat]].

[23] J. W. Chen, T. Ishikawa, L. Jin, H. W. Lin, Y. B. Yang, J. H. Zhang and Y. Zhao, "Parton Distribution Function with Non-perturbative Renormalization from Lattice QCD," arXiv:1706.01295 [hep-lat].

[24] H. W. Lin, J. W. Chen, T. Ishikawa and J. H. Zhang, "Improved Parton Distribution Functions at Physical Pion Mass," arXiv:1708.05301 [hep-lat].

[25] X. Xiong, X. Ji, J. H. Zhang and Y. Zhao, "One-loop matching for parton distributions: Nonsinglet case,” Phys. Rev. D 90, no. 1, 014051 (2014) doi:10.1103/PhysRevD.90.014051 [arXiv:1310.7471 [hep-ph]].

[26] Y. Q. Ma and J. W. Qiu, "Extracting Parton Distribution Functions from Lattice QCD Calculations," arXiv:1404.6860 [hep-ph].

[27] T. Ishikawa, Y. Q. Ma, J. W. Qiu and S. Yoshida, "Practical quasi parton distribution functions," arXiv:1609.02018 [hep-lat].

[28] J. W. Chen, X. Ji and J. H. Zhang, "Improved quasi parton distribution through Wilson line renormalization,” Nucl. Phys. B 915, 1 (2017) doi:10.1016/j.nuclphysb.2016.12.004 [arXiv:1609.08102 [hep-ph]]. 
[29] X. Xiong, T. Luu and U. G. MeiÃßner, "Quasi-Parton Distribution Function in Lattice Perturbation Theory," arXiv:1705.00246 [hep-ph].

[30] M. Constantinou and H. Panagopoulos, "Perturbative renormalization of quasi-parton distribution functions,” Phys. Rev. D 96, no. 5, 054506 (2017) doi:10.1103/PhysRevD.96.054506 [arXiv:1705.11193 [hep-lat]].

[31] J. Green, K. Jansen and F. Steffens, "Nonperturbative renormalization of nonlocal quark bilinears for quasi-PDFs on the lattice using an auxiliary field," arXiv:1707.07152 [hep-lat].

[32] A. V. Radyushkin, "Quasi-parton distribution functions, momentum distributions, and pseudo-parton distribution functions,” Phys. Rev. D 96, no. 3, 034025 (2017) doi:10.1103/PhysRevD.96.034025 [arXiv:1705.01488 [hep-ph]].

[33] K. Orginos, A. Radyushkin, J. Karpie and S. Zafeiropoulos, “Lattice QCD exploration of pseudo-PDFs," arXiv:1706.05373 [hep-ph].

[34] J. Karpie, K. Orginos, A. Radyushkin and S. Zafeiropoulos, "Parton distribution functions on the lattice and in the continuum," arXiv:1710.08288 [hep-lat].

[35] A. V. Radyushkin, "Quark Pseudo-Distributions at Short Distances,” arXiv:1710.08813 [hep-ph].

[36] S. Capitani, "Lattice perturbation theory,” Phys. Rept. 382, 113 (2003) doi:10.1016/S0370-1573(03)00211-4 [hep-lat/0211036].

[37] G. Martinelli, C. Pittori, C. T. Sachrajda, M. Testa and A. Vladikas, "A General method for nonperturbative renormalization of lattice operators," Nucl. Phys. B 445, 81 (1995) doi:10.1016/0550-3213(95)00126-D [hep-lat/9411010].

[38] I. W. Stewart and Y. Zhao, "Matching the Quasi Parton Distribution in a Momentum Subtraction Scheme," arXiv:1709.04933 [hep-ph].

[39] C. E. Carlson and M. Freid, "Lattice corrections to the quark quasidistribution at one-loop," Phys. Rev. D 95, no. 9, 094504 (2017) doi:10.1103/PhysRevD.95.094504 [arXiv:1702.05775 [hep-ph]].

[40] R. A. Briceño, M. T. Hansen and C. J. Monahan, "Role of the Euclidean signature in lattice calculations of quasidistributions and other nonlocal matrix elements," Phys. Rev. D 96, no. 1, 014502 (2017) doi:10.1103/PhysRevD.96.014502 [arXiv:1703.06072 [hep-lat]].

[41] X. Ji, J. H. Zhang and Y. Zhao, "More On Large-Momentum Effective Theory Approach to Parton Physics,” Nucl. Phys. B 924, 366 (2017) doi:10.1016/j.nuclphysb.2017.09.001 [arXiv:1706.07416 [hep-ph]].

[42] V. S. Dotsenko and S. N. Vergeles, "Renormalizability of Phase Factors in the Nonabelian Gauge Theory,” Nucl. Phys. B 169, 527 (1980). doi:10.1016/0550-3213(80)90103-0

[43] N. S. Craigie and H. Dorn, "On the Renormalization and Short Distance Properties of Hadronic Operators in QCD,” Nucl. Phys. B 185, 204 (1981). doi:10.1016/0550-3213(81)90372-2

[44] H. Dorn, "Renormalization of Path Ordered Phase Factors and Related Hadron Operators in Gauge Field Theories," Fortsch. Phys. 34, 11 (1986). doi:10.1002/prop.19860340104

[45] J. Collins, "Foundations of perturbative QCD," (Cambridge monographs on particle physics, nuclear physics and cosmology. 32)

[46] X. Ji, P. Sun, X. Xiong and F. Yuan, "Soft factor subtraction and transverse momentum dependent parton distributions on the lattice,” Phys. Rev. D 91, 074009 (2015) doi:10.1103/PhysRevD.91.074009 [arXiv:1405.7640 [hep-ph]]. 
[47] C. Monahan and K. Orginos, "Quasi parton distributions and the gradient flow," JHEP 1703, 116 (2017) doi:10.1007/JHEP03(2017)116 [arXiv:1612.01584 [hep-lat]].

[48] C. Monahan and K. Orginos, "Finite continuum quasi distributions from lattice QCD," arXiv:1710.06466 [hep-lat].

[49] B. U. Musch, P. Hagler, J. W. Negele and A. Schafer, "Exploring quark transverse momentum distributions with lattice QCD,” Phys. Rev. D 83, 094507 (2011) doi:10.1103/PhysRevD.83.094507 [arXiv:1011.1213 [hep-lat]]. 